systematic immune reaction, as measured by erythrocyte sedimentation rate (ESR) and C-reactive protein (CRP) (4). We investigate GNLY distribution and intracellular setting in peripheral blood lymphocytes of patients with OA.

Objectives: Women with knee OA (17), and age and sex appropriated control (17) were tested. All of them signed informed consent before sampling of peripheral blood (PB).

Methods: Medical history, clinical examination, $\mathrm{X}$-ray and routine laboratory testing (ESR, CRP) were used for diagnosing OA. Peripheral blood mononuclear cells (PBMC) were isolated by gradient density centrifugation and used for multiple, simultaneous intracellular [total GNLY, 9 kDa GNLY, 15 kDa GNLY and Lysosomal-associated membrane protein-1 (LAMP-1)] and surface antigens (CD3 and CD56) detection by immunofluorescence. Data were analyzed by flow cytometry or confocal microscopy.

Results: In OA and control samples the percentage of total GNLY+ cells and GNLY expressing NK and T cells did not significantly differ and correlate with ESR and CRP. The frequency of GNLY + cells was always higher in natural killer (NK) then in T cells and $9 \mathrm{kDa}$ GNLY dominated over $15 \mathrm{kDa}$ GNLY. However, $9 \mathrm{kDa}$ GNLY co-localized more with the marker of cell degranulation, LAMP-1 in polarized granules of OA patients when compared to the control or to the $15 \mathrm{kDa}$ GNLY.

Conclusions: The increase in the expression of cytotoxic $(9 \mathrm{kDa})$ over regulatory $(15 \mathrm{kDa})$ GNLY form in PBMC and its intracellular setting in polarized exocytic granules suggests the involvement of activated $\mathrm{GNLY}^{+}$lymphocytes in the immunopathogenesis of knee OA, indispensable of ESR and CRP.

References:

[1] Yamada H, Nakashima Y, Okazaki K, et al. Preferential accumulation of activated Th1 cells not only in rheumatoid arthritis but also in osteoarthritis joints. J Rheumatol 2011;38:1569-75.

[2] Clayberger C, Finn MW, Wang T, et al. $15 \mathrm{kDa}$ granulysin causes differentiation of monocytes to dendritic cells but lacks cytotoxic activity. J Immunol 2012;188:6119-26.

[3] Pardo J, Pérez-Galán P, Gamen S, et al. A role of the mitochondrial apoptosisinducing factor in granulysin-induced apoptosis. J Immunol 2004;167:1222-9.

[4] Kehler T, Laskarin G, Massari D, et al. Possible role of granulysin in pathogenesis of osteoarthritis. Med Hypotheses 2015;85:850-3.

Acknowledgements: The experiments were financed by the University of Rijeka (No.13.06.1.1.06) and "Thalassotherapia- Opatija", Opatija, Croatia.

Disclosure of Interest: None declared

DOI: 10.1136/annrheumdis-2017-eular.5499

\section{SAT0010 THE DAMP PROTEIN S100A8/A9 IS CRUCIALLY INVOLVED IN MYELOID-DERIVED SUPPRESSOR CELL (MDSC) DIFFERENTIATION AND FUNCTION IN COLLAGEN-INDUCED ARTHRITIS}

M. Kuhlencord, Y. Kusche, S. Zenker, T. Vogl, J. Roth. Institute of Immunology, Münster, Germany

Background: Over the last years, the differentiation and activation of MDSC under tumor conditions has been studied extensively. Various tumor-derived factors have been identified in promoting the accumulation of this suppressive cell population. Recent findings determined the two alarmins S100A8 and S100A9 as important factors in the differentiation of MDSC during tumor conditions. However, little is known about the S100A8/A9 driven expansion and activation, as well as the relevance of these cells in autoimmune diseases such as rheumatoid arthritis. Objectives: We therefore analyzed the mechanisms involved in S100A8/A9 driven MDSC accumulation and their functional importance in a mouse model of rheumatoid arthritis.

Methods: To investigate the effect of S100A8/A9 on MDSC differentiation, bone marrow cells from wild type (wt), S100A9 knockout (A9ko) and S100A9 transgenic (A9tg) mice were analyzed. Accumulation of MDSC and their phenotypical characterization was performed by FACS analysis and functional characterization including arginase activity, NO- and ROS-production and T cell proliferation assays.

The role of S100A8/A9 and MDSC in arthritis was investigated using the collagen-induced arthritis (CIA) mouse model. Accumulation of MDSC in different organs was analyzed by FACS and systemic S100A8/A9 levels were measured by ELISA. Ex vivo functional analysis of purified MDSC was performed to assess the potential of these MDSC to inhibit T cell responses.

Results: Our in vitro studies reveal that, in the presence of S100A8, myeloid progenitor cells differentiate to immature cells that phenotypically as well as functionally resemble MDSC. These cells are characterized by co-expression and up-regulation of arginase activity, NO- and ROS-production, and exhibit strong suppressive effects on the proliferation of both CD4- and CD8-positive T cells. Furthermore, accumulation of MDSC by extracellular S100A8 was found to be mediated via the Toll-like receptor 4 signaling pathway. In addition, lack of intracellular S100A8/A9 results in a decreased number of MDSC as well as a reduced suppressive activity of these cells, implying a dual role of these proteins for MDSC differentiation and function.

Investigating the role of S100A8/A9 and MDSC in a mouse model of CIA, a clear correlation between disease scores, MDSC numbers and systemic S100A8/A9 levels was observed. Furthermore, disease activity was reduced in wt and A9tg mice compared to A9ko mice and was in line with an increased accumulation of
MDSC in the lymph nodes. Next to an enhanced suppressive activity of MDSC isolated from lymph nodes of wt and A9tg mice, these MDSC promoted the accumulation of regulatory T cells (Treg) whilst suppressing the differentiation of TH17 cells. In contrast, MDSC isolated from lymph nodes of A9ko mice had no effect on Treg differentiation and did not inhibit TH17 emergence.

Conclusions: Our in vitro results clearly show a S100A8/A9 dependent accumulation of cells that phenotypically as well as functionally resemble MDSC also under non-tumor conditions. In vivo data strongly support the importance of these findings. By influencing MDSC accumulation and function, S100A8/A9 is critically involved in regulating the disease outcome in rheumatoid arthritis, implying an important role of S100A8/A9 derived MDSC in regulating immune reactions during autoimmune diseases.

Disclosure of Interest: None declared

DOI: 10.1136/annrheumdis-2017-eular.6683

\section{SAT0011 THE ACTIVATION OF MUSCARINIC ACETYLCHOLINE RECEPTORS INFLUENCES THE ONTOGENY OF NEUTROPHILS}

S. Sommerlatte $^{1}$, L. Kappes ${ }^{1}$, S. Pitann ${ }^{1}$, G. Marschner ${ }^{1}$, H. Heidecke ${ }^{2}$, P. Lamprecht ${ }^{1}$, A. Mueller ${ }^{1}$, T. Lange ${ }^{1}$, O. Cabral-Marques ${ }^{1}$, G. Riemekasten ${ }^{1}$ ${ }^{1}$ Rheumatology, University of Lübeck, Lübeck; ${ }^{2}$ CellTrend GmbH, Luckenwalde, Germany

Background: There is growing evidence that nervous and immune system communicate with each other through soluble mediators. ${ }^{1}$ Immune cells such as neutrophils express muscarinic acetylcholine receptors (mAChR), which are neuroimmune receptors and highly prevalent in the nervous system. ${ }^{2}$ Aberrant neutrophil functioning plays an important role in various autoimmune diseases. Dysregulation of neutrophil immune responses such as oxidative burst and migration is one of the key mechanisms leading to tissue damage in autoimmune diseases. ${ }^{3}$ However, the impact of $\mathrm{mAChR}$ activation on neutrophils remains contradictory.

Objectives: We aimed to determine effects of muscarinic receptor activation on development and functions of neutrophils.

Methods: Neutrophils were isolated from peripheral blood of healthy donors by dextran sedimentation. After one hour in the absence or presence of the natural ligand acetylcholine (Ach) $(10 \mathrm{nM}-100 \mu \mathrm{M})$ or the muscarinic agonist oxotremorine-m (oxo-m) $(10 \mathrm{nM}-100 \mu \mathrm{M})$, neutrophil respiratory burst was analyzed by dihydrorhodamine (DHR) flow cytometry assay and migration assessed by transwell assay in response to $\mathrm{N}$-formylmethionyl-leucyl-phenylalanine (fMLP). Cells that migrated were quantified by flow cytometry. To analyze the effects mAChR activation on the development of neutrophils, HL-60 cells were incubated in the presence of DMSO $(1 \%)$, oxo-m $(100 \mu \mathrm{M})$ or DMSO plus oxo-m. After 6 days, cells were harvested and expression of maturation markers (CD15, CD63 and CD16) as well as mAChR (M1-M5) were measured by flow cytometry.

Results: We observed no effects of $\mathrm{mAChR}$ activation on the respiratory burst of neutrophils. However, both $\mathrm{ACh}$ and oxo-m inhibited neutrophil migration in a dose-dependent manner, but with peculiar differences. By increasing acetylcholine concentrations, we observed a reduction of neutrophil migration in a directly proportional manner. On the other hand, while the lowest dose (10nM) of oxo-m inhibited migration most effectively, the increase of oxo-m showed inversely proportional effects on neutrophil migration. Thus, we aimed to investigate, if the highest dose of oxo-m has a different effect on neutrophils ontogeny. In agreement with the results obtained with neutrophils, the incubation of HL-60 cells with the highest dose of oxo-m showed no effect on oxidative burst and migration and induced no changes in the expression of mAChRs (M1-M5), CD16 and CD63 in HL-60 cells. However, we observed that it resulted in significantly increased surface levels of the neutrophilic lineage marker CD15.

Conclusions: Our data indicate a differential activation of mAChR affecting different steps of neutrophil ontogeny. Considering this finding, abnormalities in the activation of muscarinic receptors as have been observed in autoimmune diseases might contribute to neutrophil dysfunction and need further investigation.

\section{References:}

[1] Blalock, J. E. The syntax of immune-neuroendocrine communication. Immunol Today 15, 504-511 (1994).

[2] Milara et al. Non-neuronal cholinergic system contributes to corticosteroid resistance in chronic obstructive pulmonary disease patients. Respiratory Research (2016) 17:145.

[3] Wright, H. L. et al. The multifactorial role of neutrophils in rheumatoid arthritis Nat Rev Rheumatol 10 593-601 (2014).

Disclosure of Interest: None declared

DOI: 10.1136/annrheumdis-2017-eular.6573

\section{SAT0012 S100A8/A9 INCREASES THE MOBILIZATION OF LY6C HIGH MONOCYTES TO THE SYNOVIUM DURING EXPERIMENTAL OSTEOARTHRITIS}

N. Cremers ${ }^{1}$, E. Geven ${ }^{2}$, A. Blom ${ }^{2}$, M. van den Bosch ${ }^{2}$, P. van der Kraan ${ }^{2}$, P. van Lent ${ }^{2}$. ${ }^{1}$ Experimental Rheumatology; ${ }^{2}$ Radboud university medical center, Nijmegen, Netherlands

Background: It is increasingly recognized that part of the pathology in osteoarthri- 
tis $(\mathrm{OA})$ is driven by a low-grade inflammation in the synovium. Pro-inflammatory cytokines released locally during $\mathrm{OA}$, such as $\mathrm{S100A8/A9}$ which are expressed for prolonged periods when compared to IL-1 $\beta$, IL- 6 , and TNF- $\alpha$, may recruit monocytes from the bone marrow (BM) to the joint. In mice, two functionally distinct monocyte populations are described: (i) pro-inflammatory Ly6 $\mathrm{C}^{\text {high }}$ monocytes; and (ii) patrolling Ly6C low monocytes.

Objectives: The objective of our study is to investigate the role of S100A8/A9 in the recruitment of the different monocyte populations during early collagenaseinduced OA (CiOA).

Methods: S100A8 was intra-articularly injected into the knee joint of naïve wild type C57BL/6 (WT) mice to investigate their direct role in recruitment of monocytes. CiOA was induced by unilateral intra-articular collagenase injection in WT, and S100A9KO mice to investigate the role of S100A8/A9 in more detail. At CiOA day 7, WT and S100A9KO mice were sacrificed together with age-matched saline-injected control mice ( $n=6 /$ group), and expression of several monocyte cell markers, chemokines, and adhesion molecules were measured in the synovium and BM, in which the cells were also analyzed by FACS. Monocytes were identified as (B220/CD90/CD49b/NK1.1/Ly6G) ${ }^{\text {low }}$ CD11 b ${ }^{\text {high }}\left(\right.$ F4/80/MHCII/CD11c) ${ }^{\text {low }}$, which were further divided into subsets based on their Ly6C expression.

Results: Injection of S100A8 into the knee joints of naïve mice led to a significantly elevated expression of monocyte-related markers (Ly6C, CCR2, and CX3CR1) and monocyte attracting chemokines (MCP-1, CX3CL1, MIP1 $\alpha$, and KC) within the synovium after 1 and 3 days, suggesting that $\mathrm{S} 100 \mathrm{~A} 8 / \mathrm{A} 9$ is directly involved in the attraction of monocytes. At CiOA day 7 in WT mice, numbers of Ly6Chigh, but not Ly6 $6 \mathrm{C}^{\text {low }}$ monocytes, were strongly increased $(7.6$-fold) in the synovium as compared to saline-injected control joints. In contrast, S100A9KO mice showed a significant increase in Ly6Clow monocytes (2-fold), whereas the number of Ly6 $\mathrm{C}^{\text {high }}$ monocytes remained unaffected. Concurrently, a strong upregulation of several chemokines (MCP1, CX3CL1, KC, and MIP1 $\alpha$ ) was observed locally in the synovium, of which only the Ly6Clow mobilization marker CX3CL1 was significantly higher in S100A9KO mice, corresponding with the increased Ly6Clow monocytes in the synovium of S100A9KO mice. This could however not explain the local increased number of Ly6Chigh monocytes at CiOA day 7 in WT mice, and therefore we next investigated the main source of the monocytes, which is the BM. We observed a decrease of $14 \%$ of myeloid cells (consisting partly of Ly6C $\mathrm{C}^{\text {high }}$ monocytes) in the BM of WT mice at CiOA day 7, whereas there were no changes in the BM of S100A9KO mice, suggesting that S100A8/A9 affects the release of myeloid populations from the $B M$. In line with that, expression of adhesion molecules (LFA-1, VCAM, VE-cadherin, PECAM1, and L-selectin) was lower at CiOA day 7 in the BM of S100A9KO mice when compared to WT mice.

Conclusions: Local induction of OA leads to S100A8/A9 production, and subsequently to the mobilization of Ly6 $6 \mathrm{C}^{\text {high }}$ monocytes into the joint, driving $\mathrm{OA}$ pathology.

Disclosure of Interest: None declared

DOI: 10.1136/annrheumdis-2017-eular.6181

\section{SAT0013 THE PATHOGENIC ROLE OF MYELOID CD141+ DENDRITIC CELLS IN INFLAMMATORY ARTHRITIS}

M. Canavan ${ }^{1}$, T. McGarry ${ }^{1}$, K. Killick ${ }^{2}$, B. Moran ${ }^{3}$, D. Cluxton ${ }^{3}$, C. Orr ${ }^{4}$, H. Convery ${ }^{4}$, S. Wade ${ }^{1}$, S. Wade ${ }^{1}$, R. Mullan ${ }^{5}$, J. Fletcher ${ }^{3}$, D.J. Veale ${ }^{4}$ U. Fearon ${ }^{1}$. 'Molecular Rheumatology, Trinity College Dublin, Dublin; ${ }^{2}$ Systems Biology Ireland, Conway Institute of Biomolecular and Biomedical Research, UCD, Dublin $4 ;^{3}$ Immune Regulation in Human Disease, Trinity College Dublin, Dublin; ${ }^{4}$ The Centre for Arthritis and Rheumatic Diseases, St Vincents University Hospital, Dublin 4; ${ }^{5}$ Rheumatology Department, The Adelaide and Meath Hospital, Dublin, Ireland

Background: Dendritic cell (DC) are a heterogeneous group of antigen presenting cells that can be subdivided into CD1c ${ }^{+} \&$ CD $141^{+}$DC. CD $141^{+}$DC are a rare population of DC that were first discovered in 2010 in human peripheral blood. Due to their rarity very little is known about the function of these cells in other tissue in or indeed disease. These newly described DC subset have thus never been described in Inflammatory Arthritis (IA) or any of the rheumatic diseases.

Objectives: To identify CD141 DC in IA synovium and functionally assess if these cells play a pathogenic role in IA.

Methods: CD141+DC were magnetically purified from synovial fluid mononuclear cells (SFMC) and peripheral blood mononuclear cells (PBMC) stimulated and stained with a panel of fluorochrome conjugated antibodies for multicolour flow cytometry. CD141+ DC isolated and purified from IA synovial fluid were subsequently cocultured with allogenic $C D 3^{+} \mathrm{T}$ cells for $6 \mathrm{~d}$ after which intracellular cytokine production was assessed by flow cytometry. Supernatants from this DC-T cell cocultures were used to treat synovial fibroblasts \& the expression of adhesion molecules, cytokines \& MMPs was measured. Finally using sorted populations of CD141+ DC from SFMC and PBMC, RNA sequencing was performed and differentially expressed genes and interaction network analysis were identified using the DeSeq2 R package, Ingenuity ${ }^{\circledR}$ Pathway Analysis (IPA) and InnateDB and Cytoscape.

Results: Within IA synovial fluid (SF), CD141+ DC are significantly enriched compared to WB \& express higher levels of the costimulatory activation markers CD80 CD86 and CD40. Following coculture of these SF CD141+ DC with CD3 ${ }^{+} \mathrm{T}$ cells, CD141 ${ }^{+} \mathrm{DC}$ induce both $\mathrm{CD} 8^{+} \& \mathrm{CD} 4^{+} \mathrm{T}$ cell proliferation. SF CD141 ${ }^{+} \mathrm{DC}$ induce Granzyme B production from $\mathrm{CD}^{+} \mathrm{T}$ cells \& TNF $\alpha$, IFN $\gamma$ \& GMCSF from
$\mathrm{CD}^{+} \mathrm{T}$ cells. The IA synovium consists of a complex interplay of multiple cell types. Therefore next we examined the effect of this CD $141^{+} \mathrm{DC}-\mathrm{T}$ cell interaction on the key invasive cells in the synovium - synovial fibroblasts. Supernatants from CD141 activated T cells were cultured with fibroblasts \& induced expression of ICAM-1, IL-6, IL-8, MMP1 \& MMP3. SF CD141 expressed significantly higher levels of the the hypoxia marker TREM1, activation of which induces further expression of CD80, CD86 and CD40. Coculture of these TREM1 activated CD141 with $\mathrm{CD}^{+} \mathrm{T}$ cells increases IFN $\gamma$ and IL-17a production. Finally RNASeq analysis revealed that there are 2089 differentially expressed genes between SF CD $141^{+}$DC \& WB CD141+DC. These genes are involved in a number of key pathways such as energy metabolism, chemokine \& cytokine signalling. Principal Component Analysis (PCA) revealed that $\mathrm{CD} 141^{+} \mathrm{DC}$ with the synovium are distinctly different from blood CD $141^{+}$DC

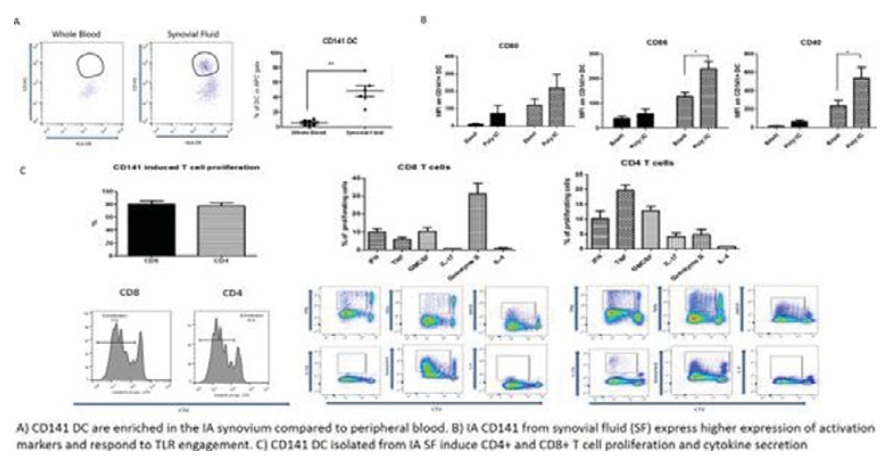

Conclusions: CD141+ DC are enriched in the IA joint in an active state. RNASeq analysis revealed they are distinct from blood $C D 141^{+} \mathrm{DC}$ and our in vitro data would support the hypothesis that these CD $141^{+} \mathrm{DC}$ contribute to synovial inflammation and joint destruction.

Disclosure of Interest: None declared

DOI: 10.1136/annrheumdis-2017-eular.6169

\section{SAT0014 THE MUTATED RNA SPLICING PROTEIN HNRNP-A3 IS A NOVEL AUTOANTIGEN IN SYSTEMIC RHEUMATIC DISEASES A LINK TO WARBURG EFFECT IN RA}

B. Marklein ${ }^{1}$, M. Hansson ${ }^{2}$, G. Steiner ${ }^{3}$, G. Burmester ${ }^{1}$, K. Skriner ${ }^{1}$. ${ }^{1}$ Department of Rheumatology and Clinical Immunology, Charité University Medicine, Berlin, Germany; ${ }^{2}$ Rheumatology Unit, Department of Medicine, Karolinska Institutet, Stockhom, Sweden; ${ }^{3} 2$ Division of Rheumatology, Department of Internal Medicine III, Medical University of Vienna, Vienna, Austria

Background: The present study was undertaken to investigate novel anti-hnRNPs autoantibodies in rheumatic diseases.

Objectives: Novel mutated hnRNP A3 was cloned out of RA synovial tissue linking it directly to Warburg effect and lactate production in RA. Increased lactate production in RA synovia and tumors is klinked to alternative splicing process from PKM1 to PKM2 hnRNPs dependent.

Methods: After immunobloting and 2D-gel-eletrophoresis of a semipurified hnRNP fraction two protein spots were sequenced and identified to be highly similar to hnRNPA3. The hnRNP A3 variants were cloned from RA synovial tissue, which identified the isoforms found on protein level. 3700 RA sera were screened for the presence of mutated anti-hnRNP A3 autoantibodies using recombinant proteins and peptides thereof. Binding of RNA to hnRNP A3 (MA3) and mutated citrullinated A3 peptides (MCA3) the epitope recognition was investigated. Expression of hnRNP A3 in synovial tissue was analysed by

Results: Autoantibodies to MA3 protein were detected in $13 \%$ of RA ( $n=215)$ patients, in $9 \%$ SLE $(n=154)$, in $27 \%$ of MCTD patients $(n=44 / 10)$ and in less than $5 \%$ of 129 patients with other rheumatic disorders but not at all in healthy controls on immunoblot. When using renaturated MA3 on ELISA $22 \%$ of RA patients were detected and $87 \%$ of these patients had erosive arthritis. Same modification as in cancer cells were identified in synovial tissue and verified by MS and DNA sequencing. Using 2-3 citrullinated MCA3 peptides up to $81 \%$ of patients $(n=150)$ with established and $67 \%(n=2926)$ of patients with an early RA with a specificity of $97 \%$ were detected. In early RA $27 \%$ and $25 \%$ in established RA of CCP2 negative and $93 \%$ of CCP2 positive patients were identified.

By combining with the already established CCP2 and the new MCA3, 72\% of early patients are positive. MCA3 autoantibodies predominantly occur $(p<0.001)$ in an erosive, severe course of disease and approximately $60 \%$ of these patients receive a TNF alpha blocker within a year. MRL Lpr/lpr sera were hnRNP-A3 reactive and the antibody generation is Toll 7 and 9 dependent. Anti-hnRNP-A3antibodies are directed to conformational RNA binding epitopes. Expression of hnRNP-A3 revealed the antigen to be overexpressed in RA synovial tissue.

Conclusions: Mutated hnRNP-A3 is as a novel Toll7/9 dependent autoantigen in systemic rheumatic diseases. These mutated proteins are major components of RNA and DNA containing alternative splicing complexes leading to the Warburg effect and and autoantibodies predominantly occurre in an erosive, and severe courses of RA. 